\title{
How to build a lower-body differential pressure chamber integrated on a tilt-table: A pedagogy tool to demonstrate the cardiovagal baroreflex
}

\author{
Michael M. Tymko ${ }^{a \star}$ \\ ${ }^{a}$ Centre for Heart, Lung, and Vascular Health, School of Health and Exercise Science, Faculty of Health \\ and Social Development, University of British Columbia, 3333 University Way, Kelowna, BC V1V 1V7, \\ Canada \\ *mike.tymko@alumni.ubc.ca
}

\section{OPEN ACCESS}

\section{Citation: Tymko MM. 2016. How to build a lower-body differential pressure chamber integrated on a tilt-table: A pedagogy tool to demonstrate the cardiovagal baroreflex. FACETS I: 225-244. doi: 10.1139 / facets-20 I 6-00 I 2. \\ Editor: Steve John Alsop \\ Received: March 31, 2016}

Accepted: September 8, 2016

Published: November 30, 2016

Copyright: (c) 2016 Tymko.. This work is licensed under a Creative Commons Attribution 4.0 International License (CC BY 4.0), which permits unrestricted use, distribution, and reproduction in any medium, provided the original author(s) and source are credited.

Published by: Canadian Science Publishing

\begin{abstract}
The cardiovagal baroreflex is an important physiological reflex that is commonly taught in health-related university physiology courses. This reflex is responsible for the rapid maintenance of blood pressure through dynamic changes in heart rate (HR) and vascular resistance. The use of lower-body negative pressure (LBNP) and lower-body positive pressure (LBPP) can manipulate these stretch sensitive baroreceptors. High performance and relatively inexpensive homemade LBNP and LBPP chambers can be easily constructed providing a valuable tool for both research and teaching purposes. There has been previous documentation of how to build a LBNP chamber; however, the information available usually lacks appropriate construction details, and there is currently no literature on how to build a chamber that can accommodate both LBNP and LBPP. In addition, a recently developed novel LBNP/LBPP chamber positioned on a $360^{\circ}$ tilt-table provided the unique utility of superimposing both LBNP/LBPP and body position as independent or combined stressors to alter central blood volume. The primary purposes of this manuscript are to (1) provide step-by-step instructions on how to build a tilt-table LBNP/LBPP chamber, and (2) demonstrate the effectiveness of a tilt-table LBNP/LBPP chamber to facilitate undergraduate and graduate learning in the laboratory by effectively demonstrating the cardiovagal baroreflex.
\end{abstract}

Key words: lower-body negative pressure, lower-body positive pressure, cardiovagal baroreflex

\section{Introduction}

The cardiovagal baroreflex is a bi-directional, pressure/stretch-sensitive, negative feedback response to changes in mean arterial pressure (MAP) mediated by the autonomic nervous system (Mancia et al. 1984; Fadel and Raven 2012). This reflex is critical for moment-to-moment regulation of heart rate (HR) and vascular resistance in humans and is often a fundamental concept taught in physiology and medical-related courses. The additional value of laboratory demonstrations of physiological functions are well known (O’Donaughy et al. 2002; Berg et al. 2012), as they are critical for student learning and development in the physiological sciences.

There are several well-established methods that can be used to demonstrate baroreflex responses in a laboratory setting in humans, which include the following: (1) sitting and standing protocols 
(Zhang et al. 2009), (2) steady-state changes in body position (i.e., head-down tilt (HDT); Tymko et al. 2015), (3) thigh cuff release (Ogoh et al. 2010), (4) neck suction (Eckberg et al. 1975; Ebert et al. 1984), (5) the modified Oxford technique (Ebert and Cowley 1992), and (6) lower-body negative pressure (LBNP; Stevens and Lamb 1965). From a teaching perspective, sustained or progressive LBNP is a reliable method for eliciting a cardiovagal baroreflex response, as it is noninvasive, reproducible (Howden et al. 2001), easy to operate, and the pressure stimulus can be easily adjusted and controlled on an individual basis. LBNP experimentation requires the participant to lie in a vacuum-sealed chamber; with the use of suction provided by a vacuum source, the pressure in the LBNP chamber decreases relative to atmospheric pressure. Exposure to negative pressure results in blood translocating from the upper body into the lower extremities, causing a reduced venous return and MAP, which changes the afferent nervous outflow from baroreceptors. The baroreflex involves a cardiac and vascular arm, with responses mediated by the sympathetic and parasympathetic nervous system. The cardiac response can be easily monitored using recordings of HR and stroke volume (SV), whereas the vascular response can be monitored with measures of muscle sympathetic nervous activity (MSNA), vascular ultrasound, or both. These latter techniques, however, require expensive equipment and trained personnel. Both of these responses simultaneously contribute to the maintenance of MAP, which can be monitored on a beat-by-beat basis.

Although LBNP has been used extensively for the past several decades, there has recently been an emergence in the utility of lower-body positive pressure (LBPP), particularly for rehabilitation (Takacs et al. 2013). Unlike LBNP chambers, LBPP chambers have been employed to investigate the effects of increased venous return and MAP, which has profound effects on the cardiovascular and cerebrovascular systems (Nishiyasu et al. 2007; Perry et al. 2014). For example, LBPP chambers have been employed to investigate the regulatory responses of the brain to transient increases in blood pressure (Perry et al. 2013). Ideally, having access to both LBNP and LBPP chambers would be highly useful for demonstrating the integrated cardiovascular responses to simulated central volume loading (using LBPP) and unloading (using LBNP).

As previously mentioned, both steady-state changes in body position, such as head-up tilt (HUT) and HDT, and lower-body differential pressure (negative and positive) can have profound effects on venous return, cardiac output (CO; the product of HR and SV), and MAP (Stevens and Lamb 1965; Tymko et al. 2015). In light of this, a recently developed novel innovation is a suspended LBNP/LBPP chamber fastened to a $360^{\circ}$ tilt-table. Although there has been previous research using a combination of upright tilt and LBNP (Thomas et al. 2009; Deegan et al. 2010), this apparatus is unique as it allows the manipulation of HUT and HDT concomitantly with LBNP or LBPP. As such, the apparatus has great utility as a research tool to improve the current understanding of the mechanisms involved in blood pressure regulation, as well as being a valuable teaching instrument.

Lower-body differential pressure chambers can be purchased commercially; however, these are usually expensive (US $\$ 10,000$ to $\$ 20,000$ ) and have a similar performance as homemade chambers, which are relatively easy to construct. Helpful documentation on how to construct a basic LBNP chamber exists (Esch et al. 2007; refer to Supplementary Material 1); however, other literature has lacked an appropriate amount of detail (Lategola and Trent 1979; Verghese and Prasad 1993; Hisdal et al. 2003; Russomano et al. 2005). In addition to LBNP, the use of LBPP can be beneficial for showing the bi-directionality of the cardiovagal baroreflex. Yet, there are no published details on how to build a chamber that can accommodate both LBNP and LBPP, nor one that combines LBNP/LBPP and steady-state tilt. Ideally, a constructed lower body differential pressure chamber should be able to achieve both negative pressures and positive pressures while having a capacity to manipulate body 
position, to maximize research and teaching potential. The purpose of this manuscript is as follows: (1) for the first time, to outline a strategy to construct a LBNP/LBPP tilt-table apparatus, and (2) to demonstrate how this apparatus can be effectively used in the laboratory setting for teaching purposes using sample data traces.

After building a LBNP/LBPP tilt-table apparatus, the students (undergraduate and (or) graduate students) will accomplish the following learning objectives after conducting the proposed laboratory exercises: (1) be able to describe the specific cardiovagal baroreflex-mediated mechanisms that are responsible for the maintenance of blood pressure during LBNP and LBPP in supine position; (2) be able to recognize cardiovascular collapse, a potential response when using LBNP, to ensure participant safety; (3) be able to describe the specific cardiovagal baroreflex-mediated mechanisms that are responsible for blood pressure regulation during changes in body position (e.g., $45^{\circ} \mathrm{HDT}$, supine, and $45^{\circ} \mathrm{HUT}$ ) at rest; and (4) understand the differences in cardiovascular response to LBNP in different body positions.

\section{Materials and methods}

\section{Overview}

The additional value of the laboratory demonstrations of physiological functions are well known (O'Donaughy et al. 2002; Berg et al. 2012), as they are critical for student learning and development in the physiological sciences. However, the use of LBNP or LBPP is usually restricted to research purposes, and its use as a teaching instrument is often overlooked. Herein, we provide an example of how the constructed chamber (see Results section for construction details) can be used as a demonstration tool to aid undergraduate student learning of the cardiovagal baroreflex, and we quantify the effectiveness of the laboratory demonstration with an anonymous, voluntary student survey. Laboratory demonstrations involving LBNP or LBPP or both should be performed under supervision by experienced and knowledgeable personnel. Improper use of LBNP/LBPP could lead to participant injury.

\section{Ethical approval}

Appropriate ethical approval is always needed to perform laboratory demonstrations using human participants. The following experimental protocols were approved by a Human Ethics Committee at the University of British Columbia and were in accordance with the Canadian Government TriCouncil Policy Statement on Human Research (TCPS2) and the Declaration of Helsinki.

\section{Participant considerations}

The recruited male participant (height, $177 \mathrm{~cm}$; weight, $88 \mathrm{~kg}$ ) filled out a health history questionnaire to ensure normal pulmonary, cardiovascular, and cerebrovascular health. Participant exclusion criteria included low resting blood pressure (e.g., $<100 \mathrm{~mm} \mathrm{Hg}$ systolic blood pressure and $<60 \mathrm{~mm} \mathrm{Hg}$ diastolic blood pressure), and (or) a history of syncope (i.e., fainting), as these participants are at greater risk of reaching cardiovascular collapse during LBNP. Participants were also non-smokers, had no reported previous history of respiratory, cardiovascular, or cerebrovascular diseases, and were not taking any medications. In addition, the participant was asked to refrain from vigorous physical activity, alcohol consumption, and caffeine for at least $12 \mathrm{~h}$ prior to experimentation.

\section{Participant instrumentation}

The participant placed a kayak skirt, which was integrated with the lid of the chamber, around the iliac crest and positioned themselves within the chamber in the upright (i.e., 90 $0^{\circ} \mathrm{HUT}$ ) position. Improper positioning of the kayak skirt can influence the cardiovascular responses observed during LBNP and LBPP (Goswami et al. 2008). Once proper positioning of the kayak skirt was attained, 
two velcro stretchable waist belts were fastened around the participant to ensure an air-tight seal around the waist. Briefly (approximately 30 seconds), the vacuum was turned on to -50 , and then $+40 \mathrm{~mm} \mathrm{Hg}$, to familiarize the participant with LBNP and LBPP stress.

\section{Cardiovascular measurements}

Cardiovascular measurements were collected at $200 \mathrm{~Hz}$ using an analog-to-digital converter (Powerlab/16SP ML880; AD Instruments; ADI; Colorado Springs, Colorado, USA). Participants were instrumented with electrocardiogram (ECG) electrodes in lead II configuration in conjunction with a bioamp (ML132; ADInstruments, Colorado Springs, Colorado, USA) to derive instantaneous HR from the R-R interval of the ECG. Beat-by-beat arterial blood pressure, CO, and SV were measured using finger photoplethysmography (Finometer Pro, Finapres Medical Systems, Amsterdam, the Netherlands). Prior to baseline data collection, the Finometer was calibrated using the returnto-flow function, and blood pressure accuracy was confirmed with manual sphygmomanometry. MAP was calculated by taking the mean of each individual arterial waveform outputted from the Finometer.

\section{Experimental protocols}

Three protocols were utilized to effectively demonstrate the cardiovagal baroreflex in one participant: (1) LBNP vs. LBPP protocol, (2) body position protocol, and (3) LBNP in different body positions (i.e., $45^{\circ} \mathrm{HDT}$, supine, and $45^{\circ} \mathrm{HUT}$ ) protocol. The following protocol termination guidelines were followed to ensure participant safety: (1) the participant voluntarily terminated the test due to the onset of subjective symptoms (e.g., gray-out, nausea, dizziness, or discomfort), or (2) the participant reached presyncope, which was identified in real time by the investigators by the sudden onset of bradycardia, and (or) a 30\% reduction of baseline systolic blood pressure (calculated in advance).

\section{Protocol \# I-LBNP and LBPP}

After instrumentation setup, the participant was instructed to lie motionless in supine position and to breathe normally for approximately $10 \mathrm{~min}$ to ensure that orthostatic-related changes in blood volume distribution were stable. Afterward, an initial 5 min quiet baseline period was recorded in supine position, and then the pressure within the chamber was immediately lowered to $-50 \mathrm{~mm} \mathrm{Hg}$ (LBNP) for $10 \mathrm{~min}$. After exposure to $10 \mathrm{~min}$ of LBNP, the vacuum was disengaged and the participant rested quietly for approximately $10 \mathrm{~min}$ of recovery. Immediately after the $10 \mathrm{~min}$ recovery period, the pressure within the chamber was increased to $+40 \mathrm{~mm} \mathrm{Hg}$ (LBPP) for $10 \mathrm{~min}$.

\section{Protocol \#2-Body position}

After instrumentation setup, the participant was instructed to lie motionless in supine position and to breathe normally for approximately $10 \mathrm{~min}$ to ensure that orthostatic-related changes in blood volume distribution were stable. Immediately afterward, $10 \mathrm{~min}$ of normal resting data were collected. This protocol was repeated in two other randomized body positions: $45^{\circ} \mathrm{HDT}$ and $45^{\circ} \mathrm{HUT}$.

\section{Protocol \#3-LBNP in different body positions}

After instrumentation setup, the participant was instructed to lie motionless and to breathe normally for approximately $10 \mathrm{~min}$ to ensure that orthostatic-related changes in blood volume distribution were stable. The protocol consisted of an initial 5 min quiet baseline period in the supine position, after which the pressure inside the LBNP chamber was immediately lowered to $-50 \mathrm{~mm} \mathrm{Hg}$ (LBNP) for $10 \mathrm{~min}$. Immediately after LBNP termination, the participant completed a 10 min recovery period and then repeated the same protocol ( $5 \mathrm{~min}$ baseline, LBNP, and recovery period) in the remaining two randomized tilt positions: $45^{\circ} \mathrm{HDT}$ and $45^{\circ} \mathrm{HUT}$. 


\section{Student survey}

To quantify the effectiveness of the laboratory demonstration for undergraduate student learning of the cardiovagal baroreflex, an anonymous survey was conducted at the end of the laboratory session. The following criteria were evaluated: (1) The LBNP demonstration aided my learning; (2) I have a better understanding of key cardiovascular physiology concepts from the LBNP demonstration; (3) The LBNP demonstration encouraged me to think critically about cardiovascular physiology, and cardiovascular collapse; and (4) I recommend the implementation of the LBNP demonstration in future classes. The students were asked to rate the usefulness of each of the criteria from 1 to 10 , with 1 being considered "not useful" and 10 being "very useful."

\section{Data analysis}

All recorded cardiovascular data were exported from our data acquisition software (ADI LabChart Pro software V7.2), and averaged into time bins (15 and $30 \mathrm{~s}$ ) using Microsoft Excel. The averaged data \pm SEM were plotted using SigmaStat (Systat, Chicago, Illinois, USA, V11.5).

\section{Results}

\section{Chamber construction}

There are several different lower-body differential chambers that are in use today, each with a unique design. The following section provides information on the vacuum source and variable transformer required, along with instructions on how to construct a LBNP/LBPP chamber integrated on a tilt-table.

\section{Vacuum source}

A commonly used inexpensive device to manipulate pressure within a differential pressure chamber is an industrial vacuum. For the chamber described below, a $120 \mathrm{~V}$, powerful industrial vacuum (6 hp) with a 12 amperage rating was used. This vacuum provided a pressure range of approximately -90 to $+40 \mathrm{~mm} \mathrm{Hg}$ with our air-tight chamber (Tymko et al. 2015), and other research groups found a similar performance with this type of vacuum (Esch et al. 2007). Real-time differential pressure was monitored during experimentation using a digital manometer (DigiMano 1000, 200-200IN, Netech Corporation, Farmingdale, New York, USA). This range of differential pressure is adequate for both laboratory demonstrations and for research projects (for a detailed review see Goswami et al. 2008).

\section{Variable transformer}

Some commercial vacuums have power settings, allowing the user to change the power output, but a more versatile way to incrementally change vacuum power output is through a variable transformer. A variable transformer provides the operator with the unique advantage of fine-tuning the pressure stimulus on an individual basis. The variable transformer had the following criteria: (1) appropriate electrical current rating of 15 amperes, (2) input voltage rating of 120 , and (3) the ability to control power output on a volt-by-volt basis. For this chamber, we used a $120 \mathrm{~V}$ input, $0-140 \mathrm{~V}$ output variable transformer (Variac, 3PN1510B, ISE, Inc., Cleveland, Ohio, USA).

\section{LBNP/LBPP chamber and tilt-table design}

The design of the LBNP/LBPP chamber and tilt-table will be presented in the following order: (1) supporting frame, (2) LBNP/LBPP chamber inner frame, (3) LBNP/LBPP outer frame, and (4) opening for the participant. For a summary of all tools required for construction, please see Table 1.

Building a structurally sound support frame is essential to ensure participant safety; however, due to the size of the supporting frame, it must be collapsible for transportation. To achieve this, three 
Table 1. List of tools required to construct the tilt-table LBNP/LBPP chamber.

\begin{tabular}{|c|c|}
\hline Tool & Description \\
\hline Metal saw & Used during the cutting of the C-channel support frames \\
\hline $\begin{array}{l}\text { Metal inert gas } \\
\text { (MIG) welder }\end{array}$ & For welding the C-channel support frames together \\
\hline Angle grinder & Only used for cleaning the metal surfaces of the C-channel support frames before welding \\
\hline Hand drill & Used for drilling in wood screws and drilling through the holes in the metal support frame \\
\hline Table saw & Used to cut through the $3 / 4$-in. plywood (outer panels) and inner frame lumber \\
\hline Silicon & Used along all edges of the chamber to provide an air-tight seal \\
\hline Jig saw & $\begin{array}{l}\text { Used to cut the hole in the top outer panel for the kayak skirt and to cut the semi-circular } \\
\text { end of the table piece }\end{array}$ \\
\hline
\end{tabular}

separate metal frames were constructed. Each of these frames were built in the same fashion, and consisted of two pieces of 48-in. long, 3 -in. wide $\times 1$-in. C-channel steel uprights connected by one 50-in. long, 3-in. $\times 3$-in. rectangular steel tube (see Fig. 1A). Each of the three supporting frames were welded together. After welding these individual supporting frames together, they were connected using four $3 \frac{112-\text { in. }}{} \times 3^{1 / 2}-$ in. pieces of wood (two on each side-only left side shown in Fig. 1A), each being 54 -in. in length. The wood braces and metal frames were joined together using 6-in. long, $1 / 2$-in. diameter, hex bolts with $1 / 2$-in. washers and $1 / 2$-in. nuts (see Fig. $1 \mathrm{~A}$ ). Once the supporting frame structure was complete, two $13 / 4$-in. diameter holes were drilled near the top of the middle metal frame to allow for a 60 -in. long, $1 \frac{112}{2}$-in. diameter metal axle to be suspended across and through the holes of the supporting frame (see Fig. 1A). The LBNP/LBPP chamber and table were bolted to this axle using three 5 -in. long, $1 / 2$-in. diameter hex bolts with $1 / 2$-in. washers and $1 / 2$-in. nuts (see the next section on the chamber construction for more details on this specific step).

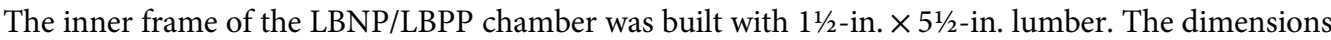
of our differential pressure chamber are 18 -in. height $\times 25$-in. width $\times 42$-in. length (see Figs. 1B, 1C). The inner frame of the LBNP chamber was fastened together using $2 \frac{1}{2}-$-in. wood screws, wherever necessary. Once the inner frame was completed, the chamber outer panels were made from $3 / 4$-in. thick plywood, and cut to size to fit over top of the inner frame. The two side plywood panels of the chamber were 18 -in. $\times 42$-in., the front panel was 25 -in. $\times 42$-in., and the bottom panel was 25 -in. $\times 18$-in. (see Figs. 1B, 1C). Once the outer panels of the chamber were cut to size, a bead of acoustic sealant (PL Acousti-Seal, Lepage, Mississauga, Ontario, Canada) was run along the inner frame before fastening the $3 / 4$-in. plywood outer panels to the inner frame.

The inner frame of the LBNP/LBPP chamber was adhered to a long table piece built out of $3 / 4-$ in . plywood that was used for the participant's upper body support. In addition, two metal supporting A-frames were bolted within the chamber to increase its performance for handling positive pressure (see Fig. 2B). The table piece was 25 -in. width $\times 75$-in. length, cut from $3 / 4$-in. plywood. The upper-most portion of the table piece (where the participant's head rests) was cut in a semi-circular shape with a jigsaw (see Figs. 1B, 1C, 2). To provide further structural support, three

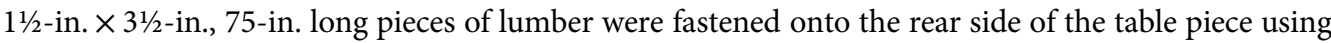
2 -in. long wooden screws: without this support the apparatus would undoubtedly break due to the overall load and weight distribution of the apparatus (the lower half being much heavier than the upper half). The chamber and tilt-table were mounted to an axle (see Figs. 1A, 2A) using three 
(A)

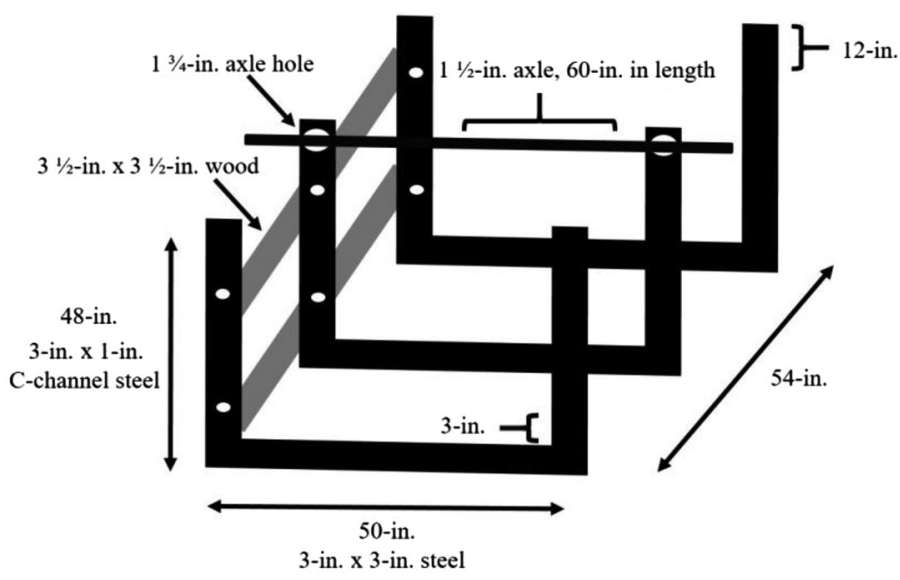

(B)

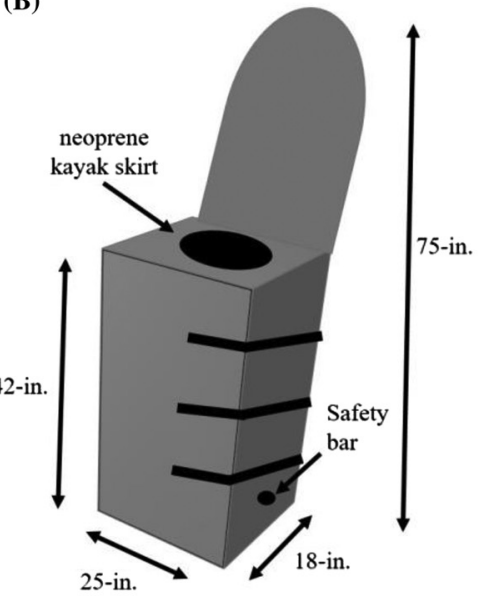

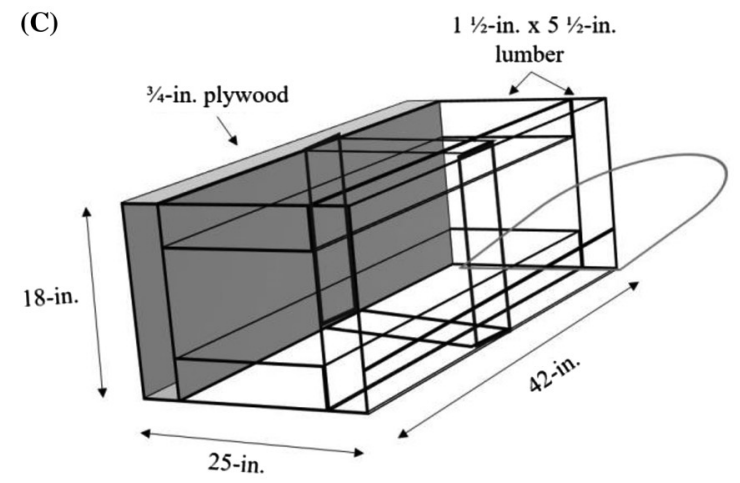

Fig. 1. Wire sketch of the tilt-table supporting frame (A) and LBNP/LBPP chamber (B and C). White dots (A) on the supporting frame represent where the 6-in. hex bolt were drilled and fastened. Note that two of the $3 \frac{1 / 2}{2}$ in. $\times 3 \frac{1}{2}-$ in. wood pieces are not represented on the right side in panel (A).

5-in. long, $1 / 2$-in. diameter hex bolts, tightened with $1 / 2$-in. nuts and $1 / 2$-in. washers. These hex bolts were inserted through $1 / 2$-in. diameter, pre-drilled holes, which were located at the mid-point of each

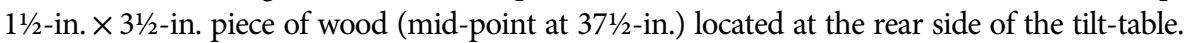

A neoprene kayak skirt (see Figs. 1, 2) was used to create an air-tight seal between the participant and the chamber. The neoprene kayak skirt was fastened inside a 17-in. in diameter hole cut within the top outer panel of the chamber (18-in. $\times 25$-in.). To fasten the neoprene kayak skirt to the chamber, a combination of heavy duty staples and construction adhesive was used. Once the last outer panel (with kayak skirt) was fastened to the chamber frame, silicon sealant was applied to the inside perimeter of the panel. The negative pressure itself causes the kayak skirt to collapse upon itself, creating a seal. Nevertheless, if the neoprene skirt fails to seal properly, or is too big, a high potential exists for the chamber to equilibrate with the atmospheric pressure, reducing the intentional stimulus. The outer (top) plywood panel containing the neoprene kayak skirt (see Figs. 1B, 2) was built so that it was removable, allowing the participant to enter the kayak skirt before entering the chamber while being in the upright position.

After the participant is placed inside the chamber (see Fig. 2B), the front outer panel of the chamber was able to shut tightly using ratchet straps. An air-tight seal between the front outer panel of the 
(A)

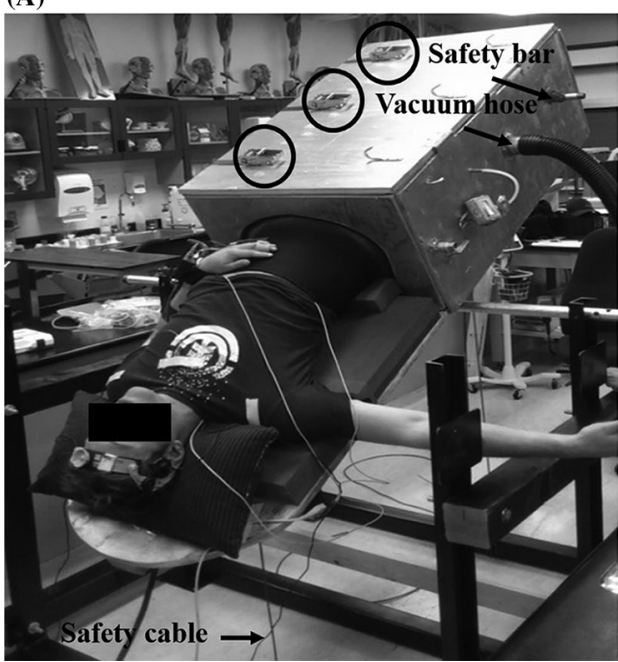

(B)

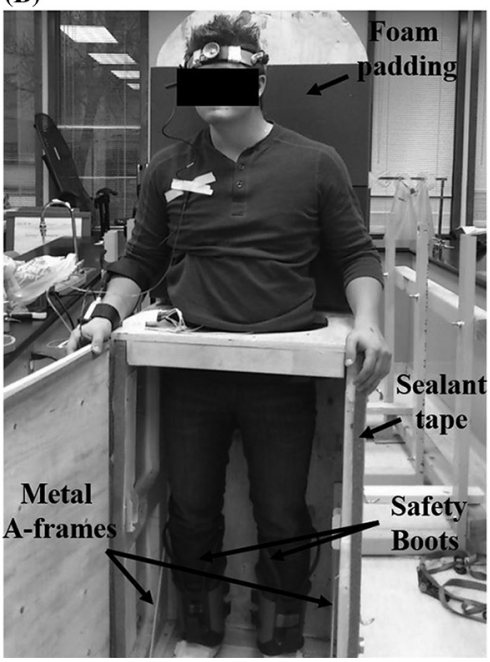

Fig. 2. Picture of the LBNP/LBPP chamber with tilt-table during a HDT LBNP protocol (A) and the inside of the LBNP/LBPP chamber (B). The three black circles highlight the three ratchet straps that were used to tighten the front chamber door to the chamber frame.

chamber and the rest of the chamber was created using adhesive sealant tape (see Fig. 2B). After the LBNP/LBNP chamber was complete, it was attached to the metal axle from the support stand using three 5 -in. long, $1 / 2$-in. diameter hex bolts with $1 / 2$-in. nuts and $1 / 2$-in. washers. The current dimensions provided for the LBNP/LBPP tilt-table chamber allowed for participants to be placed in any HUT and HDT body position desirable (see Figs. 1, 2). Changing the body position of the chamber is done manually, and the chamber is held in place using steel chain or cable connected from the chamber to the bottom of the supporting frame (see Fig. 2A).

\section{Additional features}

Importantly, participants who are placed in HDT positions with this apparatus risk falling out of the chamber. To prevent this, a 30-in. long, 1-in. diameter safety bar was placed through the bottom half of the chamber, which allowed the participant to hook into the axle using specifically designed boots (Teeter Hang-ups, Tacoma, Washington, USA) (see Figs. 2A, 2B). This served not only as a safety feature, but also held participants in position during LBPP. In addition to this feature, it is also recommended to add padding (i.e., foam or blanket) to the table portion of the chamber to aid with subject comfort (see Fig. 2B).

\section{Experimental results}

\section{LBNP and LBPP protocol}

Figure 3 illustrates the cardiovascular responses to $-50 \mathrm{~mm} \mathrm{Hg} \mathrm{LBNP}$ and $+40 \mathrm{~mm} \mathrm{Hg} \mathrm{LBPP}$ in one participant. During LBNP, HR progressively increased, while SV progressively decreased. This resulted in CO being rather stable during the beginning of the LBNP protocol, but as LBNP progressed, CO decreased near the end of LBNP protocol. Regardless of these cardiovascular changes during LBNP, MAP remained relatively unchanged during the entire LBNP protocol. During LBPP, HR, SV, and CO remained similar throughout the entire protocol; however, as expected, MAP increased by $\sim 10 \mathrm{~mm} \mathrm{Hg}$. Figure 4 represents data from an individual who reached presyncope during $-50 \mathrm{~mm} \mathrm{Hg}$ LBNP, and the protocol was terminated using our 

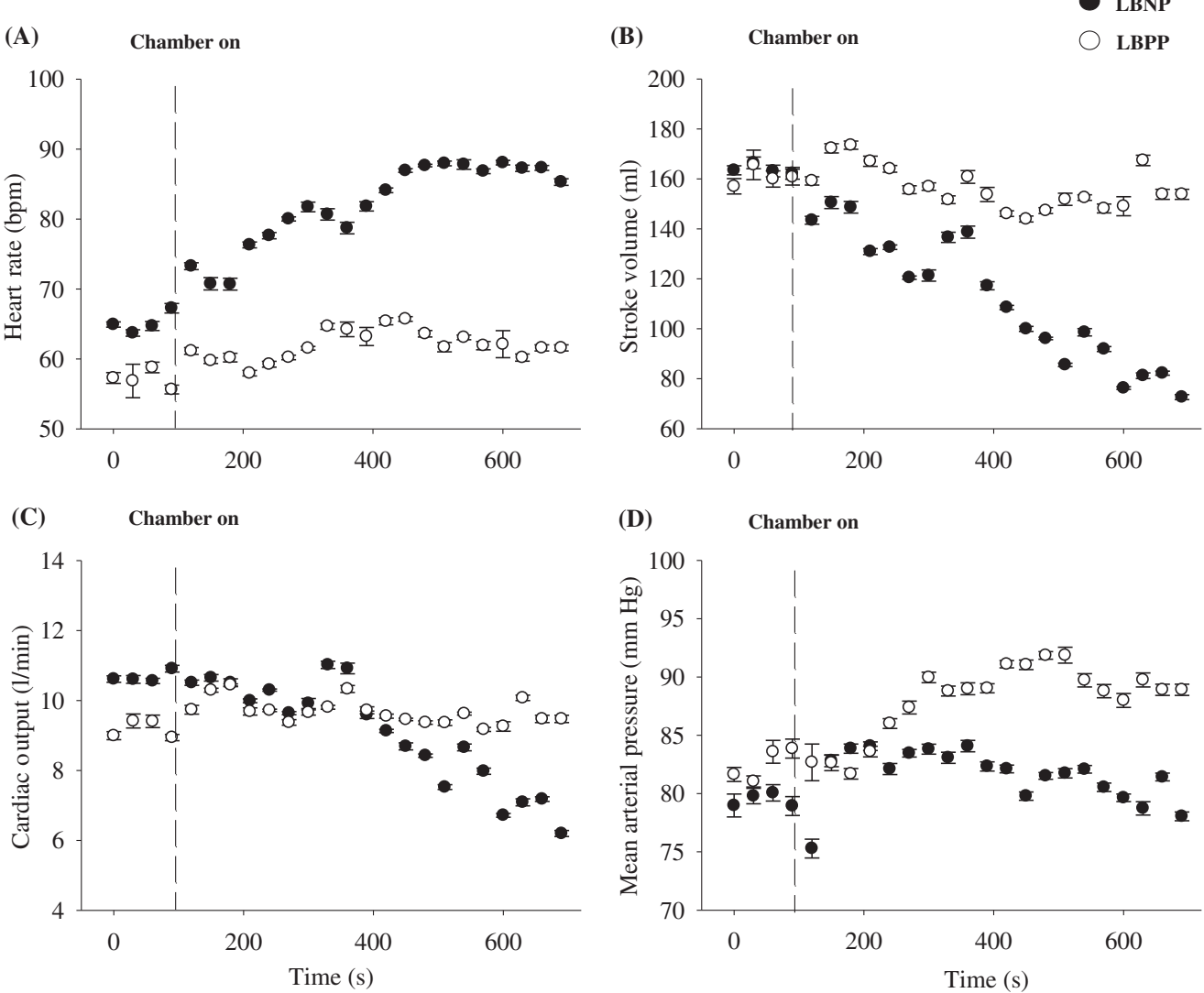

Fig. 3. Raw data tracing of the cardiovascular responses during $-50 \mathrm{~mm} \mathrm{Hg} \mathrm{LBNP}$ and $+40 \mathrm{~mm} \mathrm{Hg}$ LBPP in one subject. The first dashed line represents the initiation of lower-body differential pressure, the second dashed line represents the termination of lower-body differential pressure, on each plot. Each data point represents a $30 \mathrm{~s}$ average \pm SEM. Each protocol consisted of a 2 min eupneic breathing baseline period; immediately after baseline, the vacuum pump was turned on and pressure was sustained for a total of $10 \mathrm{~min}$. After $10 \mathrm{~min}$ of lower-body pressure stimulus, the vacuum was turned off allowing for the chamber to equilibrate with atmospheric pressure.
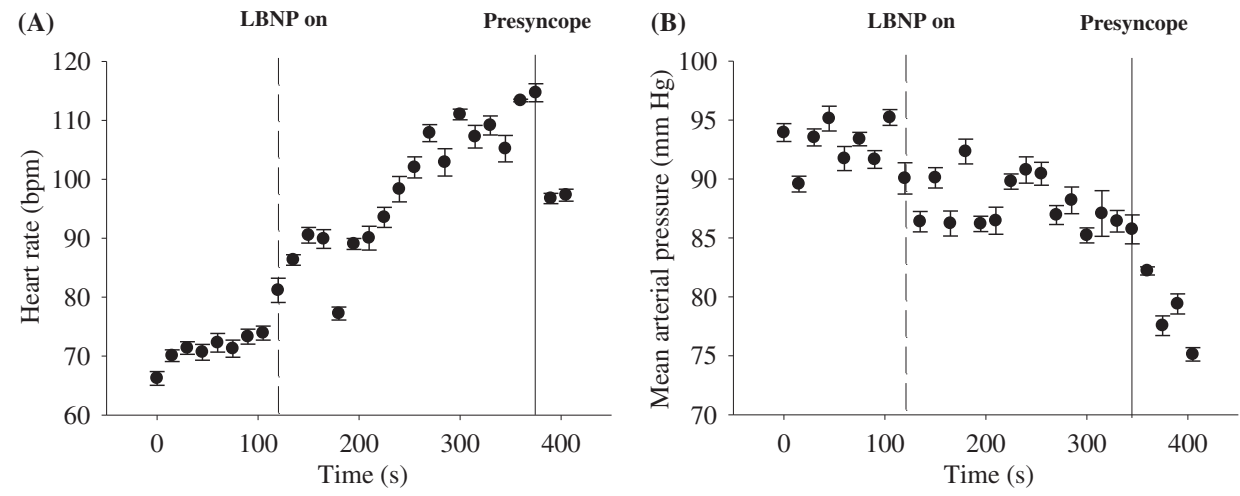

Fig. 4. Raw data tracing of one participant reaching presyncope during $-50 \mathrm{~mm} \mathrm{Hg} \mathrm{LBNP}$ in $45^{\circ} \mathrm{HUT}$ position. The dashed line represents the initiation of lower-body negative pressure, the solid line represents the termination of lower-body differential pressure due to the participant reaching presyncope. Each data point represents a $15 \mathrm{~s}$ average \pm SEM. Each protocol consisted of a 2 min eupneic breathing baseline period; immediately after baseline, the vacuum pump was turned on to generate $-50 \mathrm{~mm} \mathrm{Hg}$ of LBNP. The protocol was terminated (i.e., vacuum pump turned off) when the participant reached cardiovascular collapse, as indicated by a relative drop in HR and MAP. 
safety guidelines (see Experimental protocols section above). The participant experienced a rapid decrease in MAP, followed abruptly by relative bradycardia.

\section{Body position protocol}

Figure 5 illustrates the cardiovascular responses to steady-state $45^{\circ} \mathrm{HUT}$, supine, and $45^{\circ} \mathrm{HDT}$ in one participant. In the $45^{\circ} \mathrm{HUT}$ position, HR and $\mathrm{CO}$ were elevated compared to supine and $45^{\circ} \mathrm{HDT}$. In contrast, SV was lower during $45^{\circ} \mathrm{HUT}$ compared to supine and $45^{\circ} \mathrm{HDT}$. As expected, in the volume loading (i.e., $45^{\circ} \mathrm{HDT}$ ) position, MAP was elevated compared to both $45^{\circ} \mathrm{HUT}$ and supine body positions.

\section{LBNP in different body positions}

Figure 6 illustrates the cardiovascular responses to steady-state $45^{\circ} \mathrm{HUT}$, supine, and $45^{\circ} \mathrm{HDT}$ combined with $-50 \mathrm{~mm} \mathrm{Hg}$ LBNP in one participant. The HR response to LBNP is dose dependent, with $45^{\circ} \mathrm{HUT}$ being the largest and $45^{\circ} \mathrm{HDT}$ being the smallest. The SV response to LBNP and body position is the opposite to HR, where the SV was reduced the most in the $45^{\circ}$ HUT position and reduced by the least amount in the $45^{\circ}$ HDT position. CO during LBNP was highest during the $45^{\circ} \mathrm{HUT}$ position, while it was similar in both supine and $45^{\circ} \mathrm{HDT}$
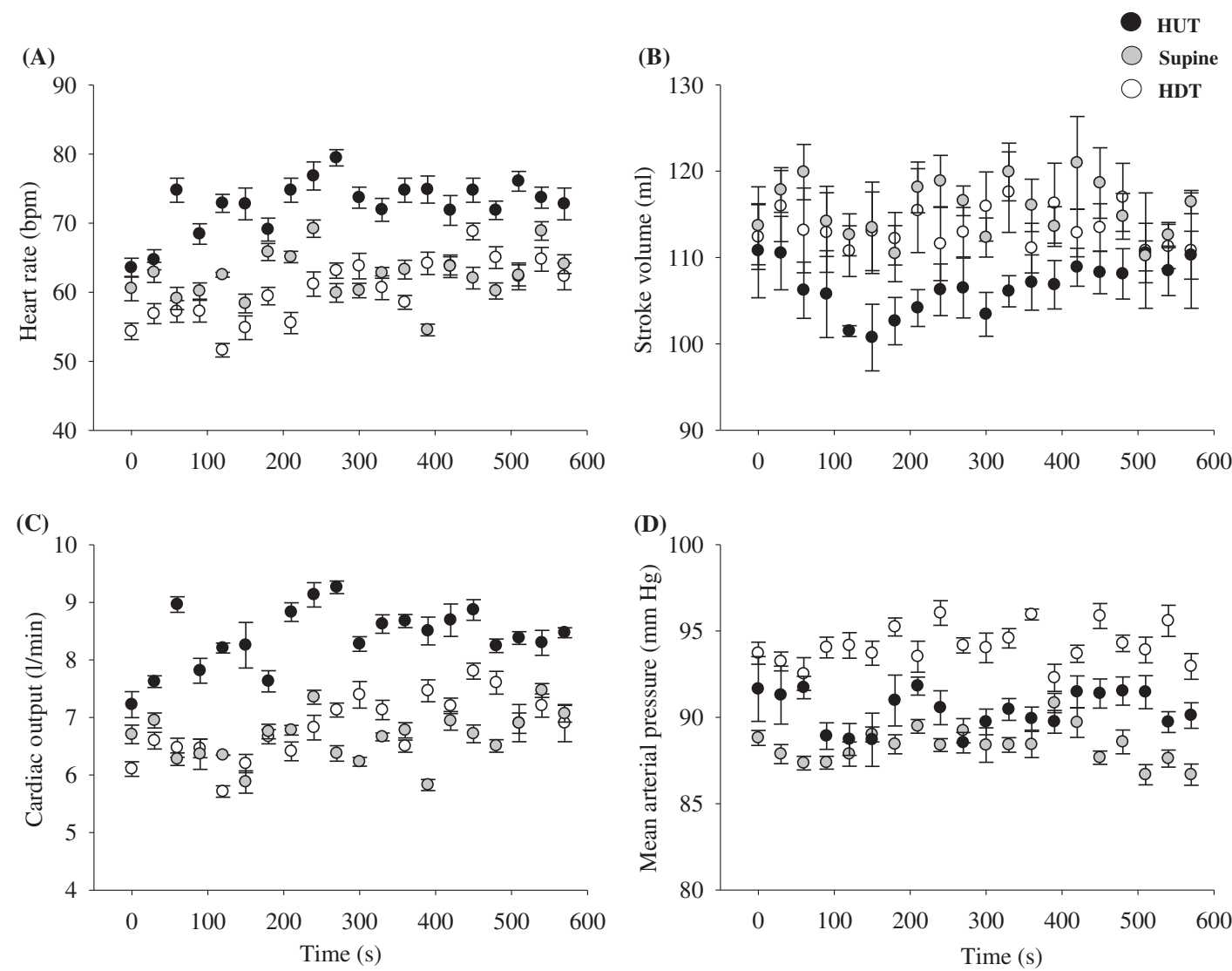

Fig. 5. Raw data tracing of the cardiovascular responses during $45^{\circ} \mathrm{HUT}$, supine, and $45^{\circ} \mathrm{HDT}$ in one subject. Each data point represents a $30 \mathrm{~s}$ average \pm SEM. Each protocol consisted of 10 min of normal eupneic breathing in each body position. 

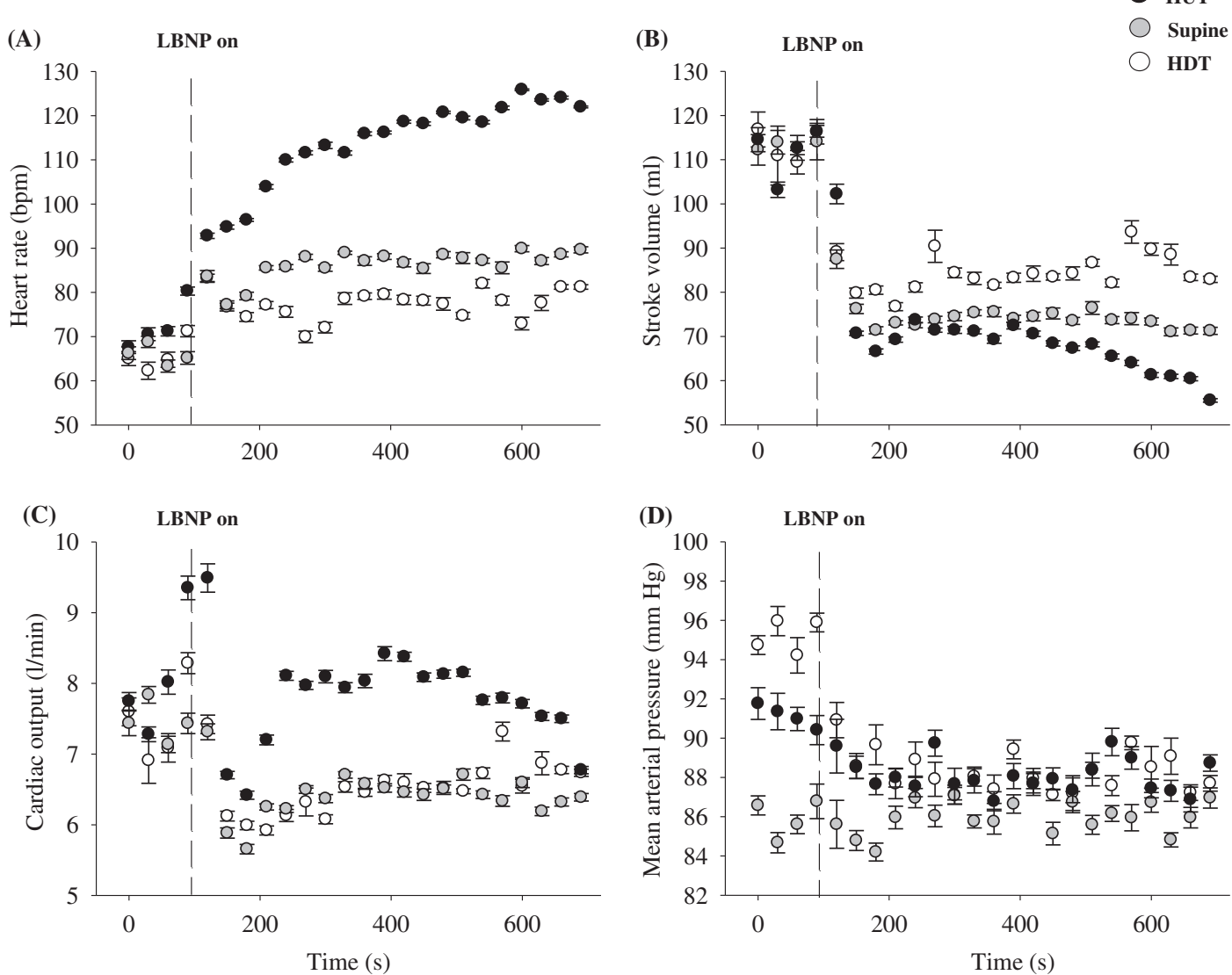

Fig. 6. Raw data trace of the cardiovascular responses during $-50 \mathrm{~mm} \mathrm{Hg} \mathrm{LBNP}$ in $45^{\circ} \mathrm{HUT}$ position, supine position, and $45^{\circ} \mathrm{HDT}$ position in one subject The dashed line represents the initiation of lower-body differential pressure, the second dashed line represents the termination of lower-body differential pressure, on each plot. Each data point represents a $30 \mathrm{~s}$ average \pm SEM. Immediately after the eupneic breathing baseline period, the vacuum pump was turned on and pressure was sustained for a total of $10 \mathrm{~min}$ in each body position. After $10 \mathrm{~min}$ of lower-body pressure stimulus, the vacuum was turned off allowing for the chamber to equilibrate with atmospheric pressure.

position. Despite different baseline MAP between body positions, MAP during LBNP was similar amongst body positions.

\section{Student survey}

Fourth year undergraduate students $(n=22)$ were present for the laboratory demonstration described above. Each of these students anonymously participated in a short written survey to provide feedback information on the effectiveness of the experimental protocol. Below are the statements provided in the survey, and the students were asked to rate the statements from 1 to 10 , with 1 being considered "not useful" and 10 being "very useful" (reports of these values are mean $\pm \mathrm{SD})$.

1. The LBNP demonstration aided my learning.

Answer: $8.0 \pm 1.7$. 
2. I have a better understanding of key cardiovascular physiology concepts from the LBNP demonstration.

Answer: $8.0 \pm 1.5$.

3. The LBNP demonstration encouraged me to think critically about cardiovascular physiology and cardiovascular collapse.

Answer: $7.8 \pm 1.8$.

4. I recommend the implementation of the LBNP demonstration in future classes.

Answer: $8.9 \pm 1.0$.

\section{Discussion}

The primary purposes of this manuscript are to (1) outline a strategy to construct a LBNP/LBPP tilttable apparatus, and (2) demonstrate how this apparatus can be effectively used in the laboratory setting for teaching purposes. After constructing a purpose-built LBNP/LBPP tilt-table apparatus, three protocols were conducted on one participant during a laboratory demonstration to undergraduate students. The laboratory demonstrations aided the students' articulation of the following learning objectives: (1) ability to describe the specific cardiovagal baroreflex-mediated mechanisms that are responsible for the maintenance of blood pressure during LBNP and LBPP in supine position; (2) ability to recognize cardiovascular collapse, a potential response when using LBNP, to ensure participant safety; (3) ability to describe the specific cardiovagal baroreflex-mediated mechanisms that are responsible for blood pressure regulation during changes in body position $\left(45^{\circ} \mathrm{HDT}\right.$, supine, and $45^{\circ} \mathrm{HUT}$ ) at rest; and (4) ability to understand why there are differences in the cardiovascular response to LBNP in different body positions ( $45^{\circ} \mathrm{HDT}$, supine, and $45^{\circ} \mathrm{HUT}$ ).

\section{Cardiovascular responses to lower-body differential pressure}

\section{Lower-body negative pressure}

The use of LBNP is an effective, non-pharmacological approach for altering central blood volume. During LBNP, blood in the upper torso is temporarily accumulated in the lower limbs, resulting in a decreased central blood volume, venous return, cardiac preload, and SV (see Fig. 3) (Cooke et al. 2004). To compensate for these physiological changes, the baroreflex is activated (sympathoexcitatory response) to increase HR and vascular peripheral resistance to maintain MAP (see Fig. 3). The magnitude of the compensatory response to LBNP is directly related to the duration of LBNP and LBNP magnitude (i.e., degree of negative pressure). For example, approximately $400-500 \mathrm{~mL}$ of blood is displaced with LBNP between -10 and $-20 \mathrm{~mm} \mathrm{Hg}$ (Thompson et al. 1990; Hanson et al. 1998), and another study concluded that -30 to $-50 \mathrm{~mm} \mathrm{Hg}$ of LBNP results in a translocation of 500$1000 \mathrm{~mL}$ of blood (Wolthuis et al. 1975). The absolute amount of blood volume translocated for a given LBNP is quite variable, and it is dependent on the effectiveness of an individual's cardiovagal baroreflex response. There is high inter-individual variability to LBNP as it can vary due to sex (Convertino 1998; Kelly et al. 2004), age (Rutan et al. 1992), height (Ludwig and Convertino 1994; Pavy-Le Traon et al. 1999), pathology (e.g., hypertension) (Kuwajima et al. 1991), and fitness level (Convertino 1987; Convertino 1993). However, after the accumulation of approximately $1000 \mathrm{~mL}$ of blood into the lower limbs during LBNP, signs of syncope are usually present (i.e., cardiovascular collapse). This occurs when the cardiovagal baroreflex-associated physiological responses are no longer able to effectively maintain MAP, resulting in hypotension (see Fig. 4). At this stage during LBNP, MAP and HR rapidly plummet due to sympathetic nervous activity withdrawal, and the individual likely experiences nausea, light-headedness, tunnel vision, and even syncope (see Fig. 4). 


\section{Lower-body positive pressure}

Similar to LBNP, LBPP is an effective experimental method for altering central blood volume; however, in contrast to LBNP, LBPP increases central blood volume by displacing fluid from the lower limbs to the central cavity (Hinghofer-Szalkay et al. 1988; Nishiyasu et al. 1998), and this results in an increase in central venous pressure, cardiac filling, and MAP (see Fig. 3) (Fu et al. 1998; Nishiyasu et al. 1998). Despite these physiological changes, CO and HR remain relatively unchanged during LBPP (see Fig. 3) (Rubal et al. 1989). However, during long durations of LBPP (e.g., >30 min), HR and CO have been shown to increase (Geelen et al. 1992). The absolute amount of blood displacement that occurs during LBPP is unclear; however, the volume of blood displaced would be inversely proportional to LBNP (as described in the section above). Similar to LBNP, the magnitude of the response to LBPP (e.g., increase in MAP) is directly related to the duration of LBPP and LBPP magnitude (i.e., degree of positive pressure). For example, +20 and $+40 \mathrm{~mm} \mathrm{Hg}$ of LBPP have been demonstrated to increase MAP by 7 and $13 \mathrm{~mm} \mathrm{Hg}$, respectively (Perry et al. 2013). Data from the laboratory demonstration (see Fig. 3) clearly show an increase in MAP with $+40 \mathrm{~mm} \mathrm{Hg}$ with a magnitude of $\sim 10 \mathrm{~mm} \mathrm{Hg}$, similar to results found by Perry et al. (2013).

\section{Cardiovascular responses to steady-state changes in body position}

Body position is often overlooked as a profound physiological stimulus; but, in fact, when moving from supine into the upright position, gravity draws a substantial volume of blood down into the lower extremities causing a decrease in central blood volume (similar effect as LBNP), whereas an increase in central blood volume occurs during HDT position (similar effect as LBPP) (BundgaardNielsen et al. 2009; Murrell et al. 2011). To compensate for changes in body position, the cardiopulmonary and arterial baroreceptors detect changes in venous return and MAP via changes in mechanical stretch and alter $\mathrm{HR}$ and vascular resistance to maintain CO, MAP, and thus, cerebral perfusion pressure (Cooke et al. 2004; Wehrwein and Joyner 2013).

Figure 5 illustrates the steady-state cardiovascular responses to body positions $\left(45^{\circ} \mathrm{HUT}\right.$, supine, and $45^{\circ} \mathrm{HDT}$ ). In the $45^{\circ} \mathrm{HUT}$ position, when central blood volume is decreased, CO and MAP are maintained by elevating $\mathrm{HR}$ to compensate for the gravity-associated decrease in venous return; thus a reduction in SV. While in the $45^{\circ} \mathrm{HDT}$ position, when central blood volume is increased, MAP is slightly elevated compared to supine and $45^{\circ}$ HUT body position (similar to LBPP), likely due to increased peripheral resistance. These results are similar to the previous findings from our research group (Tymko et al. 2015).

\section{Cardiovascular responses during LBNP in different body positions}

The tilt-table LBNP/LBPP apparatus, built by the author, has the unique advantage of eliciting a cardiovagal baroreflex in different body positions, giving undergraduate students an important demonstration of real-time integrative human physiology. As illustrated in Fig. 6, the cardiovascular responses to LBNP are dependent on body position, and this was due to the different hydrostatic pressure gradients among the three body positions. The hydrostatic pressure gradient that is important in the context of the cardiovagal baroreflex is the vertical distance between the head and the heart of the individual. Hydrostatic pressure can be calculated using the following equation:

$$
p=\rho g h \times C
$$

where $p$ is the pressure in $\mathrm{mm} \mathrm{Hg}, \rho$ (rho) is the density of blood $\left(\sim 1.04 \mathrm{~g} / \mathrm{cm}^{3}\right), g$ is the acceleration due to gravity $\left(-9.81 \mathrm{~m} / \mathrm{s}^{2}\right), h$ is the absolute height that the head is above or below the heart in meters, and $\mathrm{C}$ is the conversion factor from kilopascals to $\mathrm{mm} \mathrm{Hg} \mathrm{(7.5).} \mathrm{In} \mathrm{the} \mathrm{HUT} \mathrm{position} \mathrm{the}$ hydrostatic pressure is negative (i.e., head above the heart), meaning that the heart must overcome 

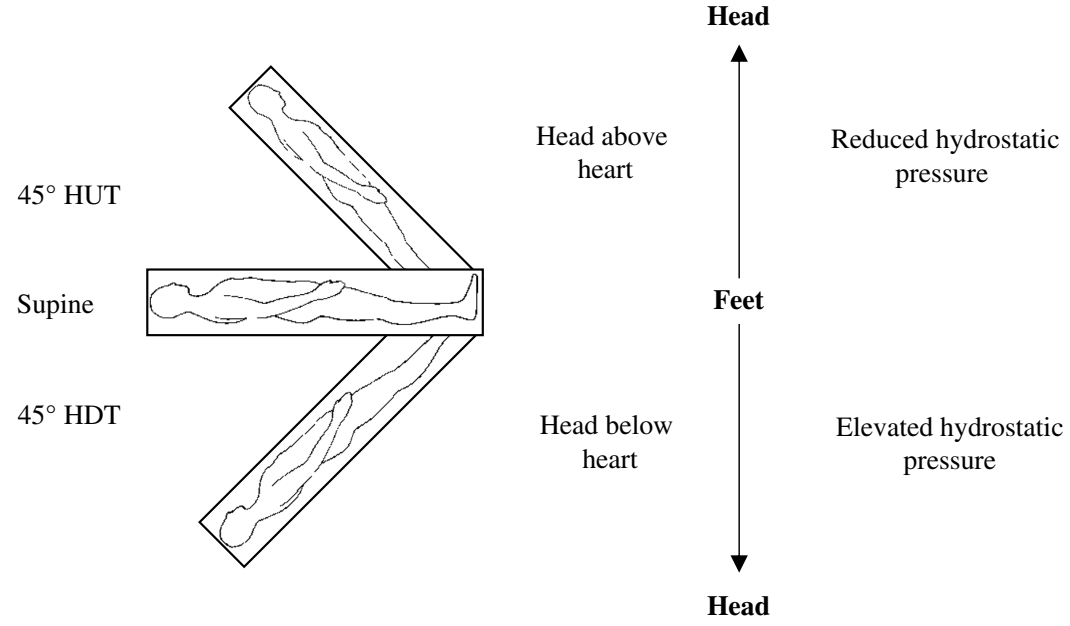

Fig. 7. Illustration of the relative hydrostatic pressure gradients present during $45^{\circ} \mathrm{HUT}$ position, supine position, and $45^{\circ}$ HDT position. The following equation can be used to calculated hydrostatic pressure: $p=\rho g h \times$ C. Assuming that the density of blood is $1.04 \mathrm{~g} / \mathrm{cm}^{3}$, and the acceleration due to gravity is constant at $-9.81 \mathrm{~m} /$ $\mathrm{s}^{2}$, the following is an example calculation of hydrostatic pressure in HUT and HDT, where the head is $0.3 \mathrm{~m}$ above or below the heart. In HUT, $p=\left(\left(1.04 \mathrm{~g} / \mathrm{cm}^{3}\right) \times\left(-9.81 \mathrm{~m} / \mathrm{s}^{2}\right) \times(0.3 \mathrm{~m})\right) \times(7.5)$, which equals $-23.0 \mathrm{~mm}$ Hg. In HDT, $p=\left(\left(1.04 \mathrm{~g} / \mathrm{cm}^{3}\right) \times\left(-9.81 \mathrm{~m} / \mathrm{s}^{2}\right) \times(-0.3 \mathrm{~m})\right) \times(7.5)$, which equals $+23.0 \mathrm{~mm} \mathrm{Hg}$. Therefore, the hydrostatic pressure is reduced in HUT $(-23 \mathrm{~mm} \mathrm{Hg})$ compared to HDT $(+23 \mathrm{~mm} \mathrm{Hg})$.

greater gravitational forces for adequate blood flow to return to the heart. In the HDT position the hydrostatic pressure gradient is positive (i.e., head below the heart), meaning that gravitational forces are aiding venous return to the heart (see Fig. 7 for an example of these calculations). Due to the reduced hydrostatic pressure associated with HUT, central blood volume is reduced compared to supine and HDT positions. This resulted in a decreased SV and elevated HR response to LBNP in HUT, compared to supine and HDT (see Fig. 6). The observed cardiac responses to LBNP are partly responsible for the maintenance of MAP; MAP is usually maintained near resting levels during LBNP (see Fig. 6).

\section{Additional physiological assessments during LBNP/LBPP}

\section{Respiratory measures}

Respiratory measurements that should be considered are respiratory flow and the end-tidal partial pressure of carbon dioxide $\left(\mathrm{P}_{\mathrm{ET}} \mathrm{CO}_{2}\right)$ and oxygen. Having these measurements could allow for the assessment of respiratory variables of the participant, as it is common to observe an increase in ventilation in response to LBNP. Respiratory gas measurements, particularly $\mathrm{P}_{\mathrm{ET}} \mathrm{CO}_{2}$, are important when measuring blood flow intracranially (see section below), as blood flow through the cerebrovasculature is sensitive to small changes in $\mathrm{P}_{\mathrm{ET}} \mathrm{CO}_{2}$. During hypercapnia (high $\mathrm{P}_{\mathrm{ET}} \mathrm{CO}_{2}$ ) blood flow through the brain increases due to downstream vasodilation of arterioles, and during hypocapnia (low $\mathrm{P}_{\mathrm{ET}} \mathrm{CO}_{2}$ ) blood flow through the brain decreases due to arteriolar vasoconstriction (Kety and Schmidt 1948). The strong effect of $\mathrm{P}_{\mathrm{ET}} \mathrm{CO}_{2}$ on cerebral blood flow is an important factor when measuring intracranial blood flow (Willie et al. 2012).

\section{Intracranial blood flow}

Transcranial Doppler (TCD) ultrasound can be employed to measure the intracranial cerebral blood flow velocity within the large conduit arteries within the cerebral vasculature. The most 
commonly insonated cerebral arteries are the middle cerebral artery (MCA; located anteriorly) and posterior cerebral artery (PCA), which supply the brain with approximately $70 \%$ and $30 \%$ of the cerebral circulation, respectively (Willie et al. 2011). The TCD system uses a $2 \mathrm{MHz}$ ultrasound probe connected to a headset, which emits sound waves through the temporal window and registers the frequency of the sound reflected from moving red blood cells within the vessel of interest. The resulting frequency shift (i.e., Doppler shift) can provide the instantaneous velocity of the moving red blood cells. Since cerebral blood flow is affected by vessel diameter, it is important for the diameter to remain constant for blood velocity to reflect the underlying changes in cerebral blood flow (Willie et al. 2011). Previous literature reports that cerebral blood velocity via TCD can be used as a surrogate measurement of cerebral blood flow during orthostatic stress (Serrador et al. 2000). During LBNP, it has been established that cerebral blood flow is reduced (Levine et al. 1994); however, during LBPP, cerebral blood flow remains relatively unchanged (Perry et al. 2013, 2014).

\section{Muscle sympathetic nervous activity}

MSNA is a direct measurement of multi-unit postganglionic activity and can obtained from fascicles of the right peroneal nerve at the popliteal fossa using a common microneurographic technique that has been used extensively since the 1960s (Vallbo et al. 2004). This technique can be time consuming and requires subject comfort to be of utmost importance. Muscle sympathetic nerve recordings provide insight into the activation of the peripheral vascular system during LBNP/LBPP. However, this technique is difficult, and it requires someone with extensive experience to be performed correctly (Vallbo et al. 2004). MSNA linearly increases with progressive LBNP until cardiovascular collapse is reached (Rea and Wallin 1989; Convertino et al. 2004), and then MSNA rapidly decreases (Sanders and Ferguson 1989; Hayoz et al. 1996), which is responsible for the decrease in peripheral vascular tone (vasodilation) and HR. During LBPP, MSNA is suppressed at positive pressures of +10 and $+20 \mathrm{~mm} \mathrm{Hg}$; however, MSNA linearly increases with more severe levels of LBPP (>30 mm Hg). Reasoning for this is currently unclear, but it is suggested that MSNA is increased due to the activation of intramuscular pressure-sensitive receptors (Fu et al. 1998).

\section{Student project application}

The tilt-table LBNP/LBPP chamber described in detail above has also been effectively used for group and (or) individual student research projects. Students developed their own experimental design, recruited appropriate participants, collected and analyzed data, wrote a lab report presenting their findings, and presented their findings to the university faculty members. Please view Supplementary Material 1 provided for the list of key references that may be useful to both students and faculty. This pedagogical approach is impactful for student learning and critical thinking, but caution must be advised when using LBNP because of the potential for eliciting adverse effects from participants (e.g., syncope, vomiting, and nausea) as illustrated in Fig. 4. It is recommended that an appropriately trained investigator be present whenever collecting data using this chamber.

\section{Conclusion}

The custom-built tilt-table LBNP/LBPP device has proven beneficial for student learning by demonstrating cardiovagal baroreflex. After completing the proposed laboratory protocols, students had a clear understanding of the following learning objectives: (1) the cardiovagal baroreflex-mediated mechanisms that are responsible for the maintenance of blood pressure during LBNP and LBPP in supine position; (2) cardiovascular collapse, a potential response when using LBNP, to ensure participant safety; (3) the cardiovagal baroreflex-mediated mechanisms that are responsible for blood pressure regulation during changes in body position 
(e.g., $45^{\circ} \mathrm{HDT}$, supine, and $45^{\circ} \mathrm{HUT}$ ) at rest; and (4) the differences in cardiovascular response to LBNP in different body positions. The cardiovagal baroreflex is an important concept taught in physiology-related courses, and demonstrating it using LBNP, LBPP, and body position in realtime aids student learning and development.

\section{Acknowledgements}

I would like to thank Drs. Philip Ainslie, Trevor Day, and Greg duManoir, along with Tony Bain, Josh Tremblay, and Ryan Hoiland, for their critical insight and aid in manuscript preparation. No funding was received for this research.

\section{Author contributions}

Conceived and designed the study: MMT. Performed the experiments/collected the data: MMT. Analyzed and interpreted the data: MMT. Drafted or revised the manuscript: MMT.

\section{Competing interests}

The author has declared that no competing interests exist.

\section{Data accessibility statement}

All relevant data are within the paper and in the Supplementary Material.

\section{Supplementary material}

The following Supplementary Material is available with the article through the journal website at doi:10.1139/facets-2016-0012.

Supplementary Material 1

\section{References}

Berg RM, Plovsing RR, and Damgaard M. 2012. Teaching baroreflex physiology to medical students: a comparison of quiz-based and conventional teaching strategies in a laboratory exercise. Advances in Physiology Education, 36: 147-153. doi:10.1152/advan.00011.2012.

Bundgaard-Nielsen M, Sørensen H, Dalsgaard M, Rasmussen P, and Secher NH. 2009. Relationship between stroke volume, cardiac output and filling of the heart during tilt. Acta Anaesthesiologica Scandinavica, 53: 1324-1328. PMID:19650800. doi:10.1111/j.1399-6576.2009.02062.x.

Convertino VA. 1987. Aerobic fitness, endurance training, and orthostatic intolerance. Exercise and Sport Sciences Reviews, 15: 223-259. doi:10.1249/00003677-198700150-00011.

Convertino VA. 1993. Endurance exercise training: conditions of enhanced hemodynamic responses and tolerance to LBNP. Medicine and Science in Sports and Exercise, 25: 705-712. doi:10.1249/ 00005768-199306000-00010.

Convertino VA. 1998. Gender differences in autonomic functions associated with blood pressure regulation. American Journal of Physiology, 275: R1909-R1920. PMID:9843880.

Convertino VA, Ludwig DA, and Cooke WH. 2004. Stroke volume and sympathetic responses to lower-body negative pressure reveal new insight into circulatory shock in humans. Autonomic Neuroscience, 111: 127-134. doi:10.1016/j.autneu.2004.02.007. 
Cooke WH, Ryan KL, and Convertino VA. 2004. Lower body negative pressure as a model to study progression to acute hemorrhagic shock in humans. Journal of Applied Physiology, 96: 1249-1261. doi:10.1152/japplphysiol.01155.2003.

Deegan BM, Cooke JP, Lyons D, Olaighin G, and Serrador JM. 2010. Cerebral autoregulation in the vertebral and middle cerebral arteries during combine head upright tilt and lower body negative pressure in healthy humans. In Proceedings of the 32nd Annual International Conference of the IEEE Engineering in Medicine and Biology Society, Buenos Aires, Argentina, 31 August-4 September 2010. pp. 2505-2508.

Ebert TJ, and Cowley AW Jr. 1992. Baroreflex modulation of sympathetic outflow during physiological increases of vasopressin in humans. American Journal of Physiology, 262: H1372-H1378. PMID:1590441.

Ebert TJ, Hayes JJ, Ceschi J, Kotrly KJ, van Brederode J, and Smith JJ. 1984. Repetitive ramped neck suction: a quantitative test of human baroreceptor function. American Journal of Physiology, 247: H1013-H1017. PMID:6507635.

Eckberg DL, Cavanaugh MS, Mark AL, and Abboud FM. 1975. A simplified neck suction device for activation of carotid baroreceptors. The Journal of Laboratory and Clinical Medicine, 85: 167-173. PMID:1141726.

Esch BT, Scott JM, and Warburton DE. 2007. Construction of a lower body negative pressure chamber. Advances in Physiology Education, 31: 76-81. doi:10.1152/advan.00009.2006.

Fadel PJ, and Raven PB. 2012. Human investigations into the arterial and cardiopulmonary baroreflexes during exercise. Experimental Physiology, 97: 39-50. doi:10.1113/expphysiol.2011.057554.

Fu Q, Sugiyama Y, Kamiya A, Shamsuzzaman AS, and Mano T. 1998. Responses of muscle sympathetic nerve activity to lower body positive pressure. American Journal of Physiology, 275: H1254H1259. PMID:9746473.

Geelen G, Arbeille P, Saumet JL, Cottet-Emard JM, Patat F, and Vincent M. 1992. Hemodynamic and hormonal effects of prolonged anti-G suit inflation in humans. Journal of Applied Physiology, 72: 977-984.

Goswami N, Loeppky JA, and Hinghofer-Szalkay H. 2008. LBNP: past protocols and technical considerations for experimental design. Aviation, Space, and Environmental Medicine, 79: 459-471. doi:10.3357/ASEM.2161.2008.

Hanson JM, Van Hoeyweghen R, Kirkman E, Thomas A, and Horan MA. 1998. Use of stroke distance in the early detection of simulated blood loss. Journal of Trauma-Injury Infection and Critical Care, 44: 128-134. doi:10.1097/00005373-199801000-00016.

Hayoz D, Noll G, Passino C, Weber R, Wenzel R, and Bernardi L. 1996. Progressive withdrawal of muscle nerve sympathetic activity preceding vaso-vagal syncope during lower-body negative pressure. Clinical Science (London), 91(Suppl.): 50-51. PMID:8813825. doi:10.1042/ cs0910050supp.

Hinghofer-Szalkay H, Kravik SE, and Greenleaf JE. 1988. Effect of lower-body positive pressure on postural fluid shifts in men. European Journal of Applied Physiology, 57: 49-54. doi:10.1007/ BF00691237. 
Hisdal J, Toska K, and Walløe L. 2003. Design of a chamber for lower body negative pressure with controlled onset rate. Aviation, Space, and Environmental Medicine, 74: 874-878. PMID:12924764.

Howden R, Tranfield PA, Lightfoot JT, Brown SJ, and Swaine IL. 2001. The reproducibility of tolerance to lower-body negative pressure and its quantification. European Journal of Applied Physiology, 84: 462-468. doi:10.1007/s004210100398.

Kelly DE, Scroop GC, Tonkin AL, and Thornton AT. 2004. Cardiovascular responses to orthostatic and other stressors in men and women are independent of sex. Clinical and Experimental Pharmacology and Physiology, 31: 50-56. doi:10.1111/j.1440-1681.2004.03949.x.

Kety SS, and Schmidt CF. 1948. The effects of altered arterial tensions of carbon dioxide and oxygen on cerebral blood flow and cerebral oxygen consumption of normal young men. Journal of Clinical Investigation, 27: 484-492. doi:10.1172/JCI101995.

Kuwajima I, Suzuki Y, Hoshino S, Shimozawa T, Kanemaru A, Sakai M, et al. 1991. Effects of aging on the cardiopulmonary receptor reflex in hypertensive patients. Japanese Heart Journal, 32: 157-166. doi:10.1536/ihj.32.157.

Lategola MT, and Trent CC. 1979. Lower body negative pressure box for $+\mathrm{Gz}$ simulation in the upright seated position. Aviation, Space, and Environmental Medicine, 50: 1182-1184. PMID:526224.

Levine BD, Giller CA, Lane LD, Buckey JC, and Blomqvist CG. 1994. Cerebral versus systemic hemodynamics during graded orthostatic stress in humans. Circulation, 90: 298-306. doi:10.1161/01. CIR.90.1.298.

Ludwig DA, and Convertino VA. 1994. Predicting orthostatic intolerance: physics or physiology? Aviation, Space, and Environmental Medicine, 65: 404-411. PMID:8024522.

Mancia G, Grassi G, Bertinieri G, Ferrari A, and Zanchetti A. 1984. Arterial baroreceptor control of blood pressure in man. Journal of the Autonomic Nervous System, 11: 115-124. doi:10.1016/ 0165-1838(84)90070-5.

Murrell CJ, Cotter JD, George K, Shave R, Wilson L, Thomas K, et al. 2011. Syncope is unrelated to supine and postural hypotension following prolonged exercise. European Journal of Applied Physiology, 111: 469-476. doi:10.1007/s00421-010-1671-8.

Nishiyasu T, Hayashida S, Kitano A, Nagashima K, and Ichinose M. 2007. Effects of posture on peripheral vascular responses to lower body positive pressure. American Journal of PhysiologyHeart and Circulatory Physiology, 293: H670-H676. PMID:17351071. doi:10.1152/ ajpheart.00462.2006.

Nishiyasu T, Nagashima K, Nadel ER, and Mack GW. 1998. Effects of posture on cardiovascular responses to lower body positive pressure at rest and during dynamic exercise. Journal of Applied Physiology, 85: 160-167.

Ogoh S, Tzeng YC, Lucas SJ, Galvin SD, and Ainslie PN. 2010. Influence of baroreflex-mediated tachycardia on the regulation of dynamic cerebral perfusion during acute hypotension in humans. Journal of Physiology, 588: 365-371. doi:10.1113/jphysiol.2009.180844.

O'Donaughy TL, Resta TC, and Walker BR. 2002. Laboratory demonstration of baroreflex control of heart rate in conscious rats. American Physiological Society, 26: 309-316. 
Pavy-Le Traon A, Louisy F, Vasseur-Clausen P, Güell A, and Gharib C. 1999. Contributory factors to orthostatic intolerance after simulated weightlessness. Clinical Physiology, 19: 360-368. doi:10.1046/ j.1365-2281.1999.00189.x.

Perry BG, Lucas SJ, Thomas KN, Cochrane DJ, and Mundel T. 2014. The effect of hypercapnia on static cerebral autoregulation. Physiological Reports, 2(6): e12059. PMID:24973333. doi:10.14814/ phy2.12059.

Perry BG, Schlader ZJ, Raman A, Cochrane DJ, Lucas SJ, and Mundel T. 2013. Middle cerebral artery blood flow velocity in response to lower body positive pressure. Clinical Physiology and Functional Imaging, 33: 483-488. doi:10.1111/cpf.12046.

Rea RF, and Wallin BG. 1989. Sympathetic nerve activity in arm and leg muscles during lower body negative pressure in humans. Journal of Applied Physiology, 66: 2778-2781.

Rubal BJ, Geer MR, and Bickell WH. 1989. Effects of pneumatic antishock garment inflation in normovolemic subjects. Journal of Applied Physiology, 67: 339-345.

Russomano T, Falcao F, Gurgel J, Piccoli L, Porto F, Dalmarco G, et al. 2005. Development of a lower body negative pressure box with an environmental control system for physiological studies. In Conference Proceedings: Annual International Conference of the IEEE Engineering in Medicine and Biology Society, Shanghai, China, September 1-4, 2005. Vol. 5. pp. 4501-4504.

Rutan GH, Hermanson B, Bild DE, Kittner SJ, LaBaw F, and Tell GS. 1992. Orthostatic hypotension in older adults. The cardiovascular health study. CHS Collaborative Research Group. Hypertension, 19: 508-519. doi:10.1161/01.HYP.19.6.508.

Sanders JS, and Ferguson DW. 1989. Profound sympathoinhibition complicating hypovolemia in humans. Annals of Internal Medicine, 111: 439-441. doi:10.7326/0003-4819-111-5-439.

Serrador JM, Picot PA, Rutt BK, Shoemaker JK, and Bondar RL. 2000. MRI measures of middle cerebral artery diameter in conscious humans during simulated orthostasis. Stroke, 31: 1672-1678. PMID:10884472. doi:10.1161/01.STR.31.7.1672.

Stevens PM, and Lamb LE. 1965. Effects of lower body negative pressure on the cardiovascular system. American Journal of Cardiology, 16: 506-515. doi:10.1016/0002-9149(65)90027-5.

Takacs J, Anderson JE, Leiter JR, MacDonald PB, and Peeler JD. 2013. Lower body positive pressure: an emerging technology in the battle against knee osteoarthritis? Clinical Interventions in Aging, 8: 983-991. doi:10.2147/cia.s46951.

Thomas KN, Cotter JD, Galvin SD, Williams MJ, Willie CK, and Ainslie PN. 2009. Initial orthostatic hypotension is unrelated to orthostatic tolerance in healthy young subjects. Journal of Applied Physiology, 107: 506-517. doi:10.1152/japplphysiol.91650.2008.

Thompson CA, Tatro DL, Ludwig DA, and Convertino VA. 1990. Baroreflex responses to acute changes in blood volume in humans. American Journal of Physiology, 259: R792-R798. PMID:2221146.

Tymko MM, Skow RJ, MacKay CM, and Day TA. 2015. Steady-state tilt has no effect on cerebrovascular $\mathrm{CO}_{2}$ reactivity in anterior and posterior cerebral circulations. Experimental Physiology, 100: 839-851. doi:10.1113/EP085084. 
Vallbo AB, Hagbarth KE, and Wallin BG. 2004. Microneurography: how the technique developed and its role in the investigation of the sympathetic nervous system. Journal of Applied Physiology, 96: 1262-1269. doi:10.1152/japplphysiol.00470.2003.

Verghese CA, and Prasad AS. 1993. Lower body negative pressure system for simulation of $+\mathrm{Gz}$ induced physiological strain. Aviation, Space, and Environmental Medicine, 64: 165-169.

Wehrwein EA, and Joyner MJ. 2013. Regulation of blood pressure by the arterial baroreflex and autonomic nervous system. Handbook of Clinical Neurology, 117: 89-102. doi:10.1016/ B978-0-444-53491-0.00008-0.

Willie CK, Colino FL, Bailey DM, Tzeng YC, Binsted G, Jones LW, et al. 2011. Utility of transcranial Doppler ultrasound for the integrative assessment of cerebrovascular function. Journal of Neuroscience Methods, 196: 221-237. doi:10.1016/j.jneumeth.2011.01.011.

Willie CK, Macleod DB, Shaw AD, Smith KJ, Tzeng YC, Eves ND, et al. 2012. Regional brain blood flow in man during acute changes in arterial blood gases. The Journal of Physiology, 590: 32613275. PMID:22495584. doi:10.1113/jphysiol.2012.228551.

Wolthuis RA, LeBlanc A, Carpentier WA, and Bergman SA Jr. 1975. Response of local vascular volumes to lower body negative pressure stress. Aviation, Space, and Environmental Medicine, 46: 697-702.

Zhang R, Claassen JA, Shibata S, Kilic S, Martin-Cook K, Diaz-Arrastia R, et al. 2009. Arterial-cardiac baroreflex function: insights from repeated squat-stand maneuvers. American Journal of Physiology-Regulatory, Integrative and Comparative Physiology, 297: R116-R123. PMID:19420293. doi:10.1152/ajpregu.90977.2008. 